\title{
Structural priming in comprehension of relative clause sentences
}

\author{
In search of a frequency $\mathrm{x}$ regularity interaction.
}

\author{
James Hutton and Evan Kidd
}

\begin{abstract}
The current chapter discusses a structural priming experiment that investigated the on-line processing of English subject and object relative clauses. Sixtyone monolingual English-speaking adults participated in a self-paced reading experiment where they read prime-target pairs that fully crossed the relativised element within the relative clause (subject versus object) across prime and target sentences. Following probabilistic theories of sentence processing, which predict that low frequency structures like object relatives are subject to greater priming effects because they are infrequent, it was hypothesised that the normallyobserved subject RC processing advantage would be eliminated following priming. The hypothesis was supported, identifying an important role for structural frequency in the processing of relative clause structures.
\end{abstract}

Just as in studies of language acquisition, the processing of relative clauses (RCs) has featured prominently as a research topic in adult psycholinguistic research. Although the two research literatures rarely overlap, they necessarily constrain each other. Sensitivity to frequency information is an important research topic in both fields, particularly those approaches that are functional or usage-based (e.g., Bates \& MacWhinney, 1989; MacDonald, Pearlmutter, \& Seidenberg, 1994; Seidenberg \& MacDonald, 1999; Tomasello, 2003). In the current chapter we discuss the role of frequency in the processing of subject and object RCs. We present the results of a structural priming study conducted with English-speaking adults that identifies a role for prior linguistic experience in the processing of these forms, suggesting that frequency of form is an important determinant of subsequent linguistic performance even in adults.

Two different classes of theories attempt to explain the processing of RCs: capacity-limit and frequency-based theories. Capacity-limit approaches argue that people possess a limited cognitive capacity that they can devote to parsing. Certain 
syntactic structures, such as object RCs, are sufficiently complex to exceed this capacity, which results in either slowed processing speed or miscomprehension (Gibson, 1998; Just \& Carpenter, 1992). On the other hand, in frequency-based theories it is argued that the processing speed and rate of comprehension accuracy of a particular syntactic structure are determined by the frequency of that structure within the language (MacDonald \& Christiansen, 2002; Wells, Christiansen, Race, Acheson, \& MacDonald, 2009). Structures that are more frequent will be processed more quickly and comprehended more accurately than structures that are less frequent. A particular research focus has been the apparent asymmetry between the processing of subject (1) and object (2) relative clause sentences.

(1) The reporter [that_attacked the senator] admitted the error.

(2) The reporter [that the senator attacked_] admitted the error.

Sentence (1) is a centre embedded subject relative clause (RC), so called because the RC (in brackets) is embedded within the main clause (the reporter admitted the error) and because the head noun the reporter occupies the subject position within the RC, as indicated by the underscore gap. In contrast, sentence (2), while also being centre embedded, is an object RC as the head noun occupies the object position within the RC, as also indicated by the underscore gap. One consistent finding in studies of RC processing is that subject RCs are read more easily than object RCs (King \& Just, 1991; Traxler, Morris \& Seely, 2002; Traxler, Williams, Blozis \& Morris, 2005). A number of capacity-limit and frequency-based theories have been developed in order to explain this phenomenon (e.g. Gibson, 1998; MacWhinney \& Pleh, 1988). In their perspective-shifting account, MacWhinney and Pleh claim that the reader always takes the perspective of the agent of a sentence. In subject RCs, the head noun is the agent throughout the sentence. However, in object RCs agency alternates between the head noun and the relative clause subject, requiring two shifts in perspective that tax the cognitive capacity of the comprehender, resulting in greater difficulty during comprehension. Other authors (e.g. Gibson, 1998; Just \& Carpenter, 1992) appeal to the word order, of RC sentences to explain this asymmetry. The non-canonical word order in object RCs (i.e. the reporter that the senator attacked...) means that the two noun phrases have to be maintained together in working memory without having been assigned thematic roles. In contrast, subject RCs have a canonical noun-verb-noun word order, which allows the head noun phrase to be assigned a thematic role before the relative clause noun phrase is encountered. In these accounts, the task of maintaining two noun phrases in working memory is argued to be more taxing than maintaining one, explaining the subject-object asymmetry.

Other capacity-limit explanations exist, but it is beyond the scope of this chapter to review them all (see Traxler et al., 2002 for an overview). The key feature that 
is common to all of these theories is that they attribute the subject-object asymmetry to the interaction of inherent properties of the sentence with the comprehender's cognitive capacity. As both these factors are static, capacity-limit theories do not predict any major role for experience in the processing of relative clause structures.

In contrast, Wells et al. (2008) and MacDonald and Christiansen (2002) have proposed a frequency-based approach to the subject-object asymmetry which predicts that processing speed for RC sentences will change with learning. The approach is based on connectionist models of language, which argue for a central role for experience in both the acquisition and processing of language (e.g., Fitz, Chang, \& Christiansen, this volume; MacDonald \& Christiansen; Seidenberg \& MacDonald, 1999; Seidenberg \& McClelland, 1989). MacDonald and colleagues claim that the difficulty ascribed to object relatives is due to that fact that ObjectSubject-Verb (OSV) word order found in object RCs is highly infrequent in English (i.e., it is irregular), whereas the SVO word order found in subject RCs is canonical (i.e., it is regular). They formalised this argument under the term 'frequency $x$ regularity' interaction.

The frequency $\mathrm{x}$ regularity argument draws inspiration from research on single word recognition (Seidenberg, 1985), where it has been shown that lexical access is determined by word frequency but mediated by orthographic regularity (i.e. the degree of correspondence between the spelling and pronunciation of a word). Speed of word recognition is more dependent on direct experience with a word when that word is orthographically irregular (e.g. pint cf. mint). When the word is orthographically regular the comprehender is able to draw on experience with lexical neighbours that rhyme with the target to aid recognition (e.g. lint and tint). For instance, the words mint, tint, and lint are orthographically regular and experience with these words will aid in the recognition and pronunciation of the word stint. However, they will not aid the recognition of the word pint, which is orthographically irregular. Speed of recognition of this word is determined by its frequency alone.

Wells et al. (2008) and MacDonald and Christiansen (2002) argued that the frequency $\mathrm{x}$ regularity approach is also applicable to syntactic constructions. That is, the frequency of a particular structure determines the processing speed of that structure, but the relationship is mediated by the structure's regularity. The more frequent the structure the more quickly it will be processed, with this effect being greater for irregular structures. Further, the effect of frequency is not proposed to be linear, but to follow the curve in Figure 1. The figure shows that processing time decreases with frequency of exposure, but asymptotes as frequency increases. 


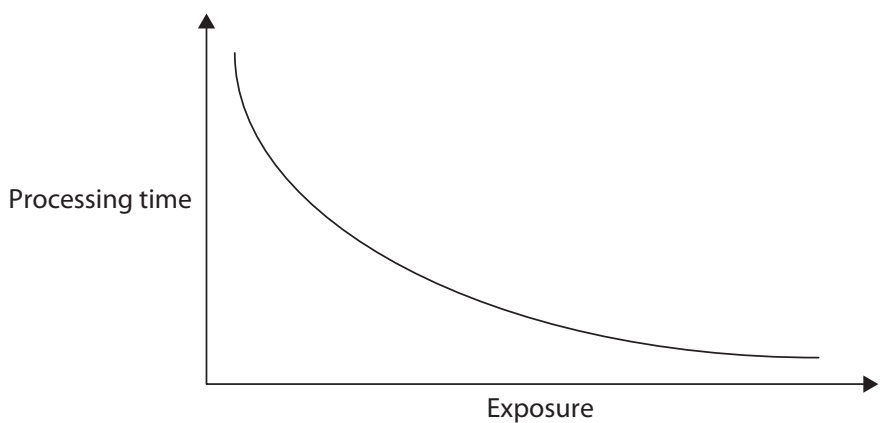

Figure 1. The theoretical relationship between exposure and processing time according to the frequency $\mathrm{x}$ regularity argument

The frequency $\mathrm{x}$ regularity argument accounts for the subject-object asymmetry, as subject RCs are both more frequent than object RCs (Roland, Dick, \& Elman, 2007), and more regular (or 'unmarked'), following the canonical word order of English. MacDonald and Christiansen's (2002) argument is therefore that the processing speed of subject RCs is determined by their frequency and the frequency of other structures that have the same SVO word order. In contrast, because object RCs have the very infrequent OSV word order, the processing speed of object RCs is determined by their frequency alone.

The crucial difference between capacity-limit theories and the frequency $\mathrm{x}$ regularity argument is that the former do not predict change in processing speed as a result of experience, whereas the latter does. The inherent syntactic complexity of a structure and a comprehender's cognitive capacity cannot be altered, and neither can the regularity of a syntactic structure. In contrast, the frequency of a structure within the language can be changed through increased exposure. In the laboratory frequency of exposure to a structure can be altered by giving participants stimulus material that contains higher or lower rates of that structure than is normal within the language. According to the frequency $\mathrm{x}$ regularity argument, high exposure to RC sentences would result in an increase in processing speed for both structures. Given that subject RCs are more frequent than object RCs to begin with, the increase in frequency will be associated with a greater increase in processing speed for object RCs than subject RCs. Further, this discrepancy in the effect of frequency will be accentuated by the effect of regularity; the effect of frequency will be greater still for object RCs because of their irregular structure. The exact magnitude of the effect of increasing exposure to RC sentences cannot be estimated using the frequency $\mathrm{x}$ regularity approach. However, it would be expected that this process would at least diminish the typical subject-object asymmetry, or perhaps eliminate it entirely. 
Wells et al. (2008) were able to eliminate the asymmetry entirely by increasing exposure to RC sentences. They conducted a training study in which participants were exposed to both subject and object RCs at four separate time points over four to eight days. Participant's online processing of subject and object RCs was measured pre- and post- training using self-paced reading. At pre-test, reading time for subject RCs was shorter than for object RCs at the main verb (the word admitted in sentences 1 and 2), the most common marker of complexity differences between subject and object RCs in the self-paced reading technique (Gibson, 1998). At post-test reading times for both structures had decreased, but the decrease was greater for object RCs so that the difference between the two structures was no longer significant. A similar decrease in reading time was found for a control group that had equal exposure to unrelated sentences. However, the interaction of exposure and structure was not evident in this group. That is, the difference between subject and object RCs was still significant at post-test. That the main effect of exposure was significant for both experimental and control groups indicates that the effect was due to a general practice effect, rather than RC-specific learning. However, the interaction of exposure with structure in the experimental group indicates that this effect was due to RC-specific learning. Further, this observation is consistent with the frequency x regularity effect. Participants received equal exposure to subject and object RCs and so the increase on the $x$-axis of Figure 1 was the same for both structures. As the relationship between exposure and processing speed is not linear, the increase in exposure was associated with a greater decrease in processing time for object RCs than for subject RCs. This was potentially because processing time of irregular object RCs is more sensitive to the effect of frequency than processing time of regular subject RCs. The difference in the effect of frequency on the processing time of subject and object RCs was sufficient to decrease processing time of object RCs to be comparable to that of subject RCs.

The findings of Wells et al. (2009) are not consistent with the capacity-limit theories, as neither the comprehender's cognitive capacity nor the structural properties of the RCs that are argued to tax that capacity could have been altered by exposure. Indeed, the cognitive capacity of the participants in the control and experimental groups was shown to be equal at both pre- and post-test, using Daneman and Carpenter's (1980) reading span task. Therefore, if cognitive capacity does affect processing then this study suggests that the effect can be overridden by learning.

\section{Structural priming}

One way of manipulating exposure to structure in the laboratory is by using the structural priming technique (for a review see Pickering \& Ferreira, 2008). Priming 
is observed in production when speakers repeat syntactic structures they have just heard; priming in comprehension is the facilitation of processing following the processing of a same-type structure. In the current chapter we report on a study that attempted to prime the comprehension of subject and object RCs in adult speakers of English. We briefly review some related research.

Traxler and Tooley (2008) primed reduced relative clause structures such as (3)-(5) in comprehension. They found that target sentences were read significantly faster than prime sentences when they contained the same subordinate verb. For example, if (3) was a prime sentence then sentence (4) was read significantly faster at the disambiguating $b y$-phrase, suggesting a priming effect. However, in cases where there was no overlap at the subordinate verb between prime and target, as between (3) and (5), there was no priming effect.

(3) The defendant examined by the lawyer was guilty.

(4) The engineer examined by the board passed with flying colours.

(5) The engineer tested by the board passed with flying colours.

Subsequent studies of comprehension have observed significant priming effects in the absence of lexical overlap between prime and target. This is important, since it is only when priming occurs in the absence of lexical overlap that we can be certain that the priming effect reflects abstract syntactic processes. For instance, Traxler (2008) observed lexically-independent priming in an eye-tracking experiment that investigated the processing of modifier-goal ambiguities (e.g., The girl tossed the blanket on the bed into the laundry). Thothathiri and Snedeker (2008) observed priming in the absence of lexical overlap in children and adults tested on the dative alternation. Finally, Nitschke (2010) successfully primed dispreferred object RC readings of ambiguous RCs in the absence of lexical overlap in L1 and L2 Germanspeaking adults and L2 Italian-speakers. The multiple observation of priming effects in the absence of lexical overlap is important because it shows that the frequency of a particular structural pattern facilitates the subsequent processing of that pattern independent of lexical content.

\section{The current study}

We now report on a study that aimed to test the frequency $\mathrm{x}$ regularity argument by investigating the processing of subject and object RCs using the structural priming method. That is, following Wells et al. (2009), we aimed to temporarily manipulate the amount of exposure participants had to both subject and object RCs. However, instead of training subjects over a period of days, we tested whether we could alter their parsing preferences within one testing session using the structural priming method. We predicted that, following the bulk of the literature 
testing RC processing, subject RCs would be easier to process than object RCs. However, following the frequency $\mathrm{x}$ regularity argument, we predicted that this subject-object asymmetry would disappear following priming.

\section{Method}

\section{Participants}

Sixty-two $(\mathrm{N}=62)$ undergraduate and postgraduate students were recruited from La Trobe University School of Psychological Science Participant Registry. All participants were native English speakers, had no known reading disability, and were aged between 18 and 35 years. Four cases ( 2 male, 2 female) were excluded from analyses because they answered less than $60 \%$ of the comprehension questions correctly. Therefore the final sample consisted of 58 participants (20 male, 38 female) with a mean age of $21.95(S D=4.12)$.

\section{Materials}

Sixty-four pairs of experimental sentences like (6) were created or taken from previous publications, where (6a) is a subject $\mathrm{RC}$ sentence and (6b) is an object RC sentence. Eight sentence pairs were adapted from King and Just (1991) and 15 were adapted from Traxler et al. (2002). The remaining 41 sentences were created anew.

(6) a. The banker that praised the barber climbed the mountain just outside of town.

(6) b. The banker that the barber praised climbed the mountain just outside of town.

Care was taken during the selection and creation of the sentences to ensure that both the head noun phrase (i.e. the banker) and the relative clause noun phrase (the barber) were equally good agents and patients for both the relative clause verb (praised) and the main clause verb (climbed). Our aim was to avoid providing semantic cues to the participant that might facilitate or interfere with sentence comprehension (e.g., Gennari \& MacDonald, 2008). The head noun and the noun within the relative clause were always animate. In general, animacy moderates the subject-object asymmetry (e.g., Mak, Vonk \& Schiefers, 2002, 2006; Traxler et al., 2002, Traxler et al. 2005), which is likely to be due to the fact that object RCs most often have inanimate head nouns. Since the current study aimed to explore the role of frequency of exposure in the subject-object asymmetry, only animate noun phrases were used. 
A further 96 filler sentences were either created or taken from previous publications. Thirty-two of the filler sentences were sentential complements such as (7), 32 were conjoined clause sentences such as (8), and the remaining 32 filler sentences were simple sentences such as (9).

(7) The general pretended that the corporal was ready to fire the weapon.

(8) The administrator admitted defeat and begrudgingly gave up his position.

(9) The snake coiled its tail.

Yes/No comprehension questions were created for all the experimental sentences and for one quarter of each category of filler sentences. For each category, including subject and object RCs, the correct answer was yes for half the questions and no for the other half. For the experimental sentences only, half of the questions interrogated the main clause and the other half interrogated the relative clause.

\section{Procedure}

Eight versions of the experiment were created. Each script had 64 experimental sentences and all of the filler sentences (160 sentences in total). Of the experimental sentences, 32 were subject RCs and 32 were object RCs, which were controlled for in word and syllable length. The experimental sentences always appeared in prime-target pairs, as shown in Table 1 . That is, there were eight object RC - object $\mathrm{RC}$ prime-target pairs, eight subject $\mathrm{RC}$ - subject RC prime-target pairs, eight object RC - subject RC prime-target pairs, and eight subject RC - object RC prime-target pairs. The order of presentation of the pairs was randomised such that no same prime-target pair type appeared in successive prime-target trials. The pairs of experimental sentences were pseudorandomly interspersed with the filler sentences, so that each group of five sentences contained one pair of experimental sentences and one of each of type of filler sentence. There was always at least one filler sentence between each pair of experimental sentences. In each script, comprehension questions were asked for 16 of the experimental sentences and 24 of the filler sentences.

We refer to those instances where the prime and target were the same structure as identity prime pairs, and the instances where the prime and target are different structures as opposite prime pairs. Each sentence also appeared once as an identity prime sentence, once as an identity target sentence, and once as opposite prime and target sentences. 
Table 1. Matrix of prime-target pair combinations

\begin{tabular}{lll}
\hline & \multicolumn{2}{c}{ Prime } \\
\hline Target & & \\
& Object RC & Subject RC \\
Object RC & Object-Object & Object-Subject \\
Subject RC & Subject-Object & Subject-Subject \\
\hline
\end{tabular}

Note. There were 8 prime-target pairs in each condition

Each participant saw one version of the experiment. The sentences were presented on a computer in a self-paced moving window design, using the E-Prime software package (MacWhinney, James, Schunn, Li, \& Schneider, 2001). The sentence initially appeared on the screen with all the letters replaced by dashes. When a target key on a button box was pressed the dashes of the first word were replaced by letters. When the key was pressed again the first word was replaced by dashes and the second word appeared. The participant continued to press the button until all words had been read, one at a time. Time taken between key presses (i.e. the time taken to read each word) was recorded. After the final word the participant was either presented with a comprehension question, which was answered yes or no by pressing keys labelled $Y$ or $N$, or a screen that read Ready for next sentence? Participants were instructed to undertake the task as quickly as possible while still comprehending the sentences, but to take whatever time they needed at the question or Ready for next sentence? screen. The next sentence began only when the participants indicated that they were ready to continue.

\section{Results}

The dependent variable was reading time at word seven (italicised in 10 and 11 as repeated below), the main verb. This follows past self-paced reading studies of subject and object relative clauses, which have shown this region to be the point at which the parser is most taxed when processing object RCs (see Gibson, 1998; Wells et al., 2009).

(10) The reporter that attacked the senator admitted the error.

(11) The reporter that the senator attacked admitted the error.

Outliers were removed from the dataset before any analyses were conducted: those values that were outside two standard deviations either side of the mean for each participant and each sentence were excluded and replaced by the participant mean for that dependent variable. This correction affected $6.7 \%$ of the data. The mean percentage 
of correct responses to comprehension questions was $81 \%$ (range: 63-100\%). Comprehension rate was higher for subject than object relatives ( $87 \%$ versus $74 \%$ ).

\section{Structure analysis}

The first set of analyses investigated whether there was a general subject-object asymmetry in the data. Only reading times of the prime sentences were included in these analyses to avoid the effect of structure being confounded by any effect of priming. Two one-way ANOVAs were conducted, one by participants $\left(F_{1}\right)$ and one by items $\left(F_{2}\right)$, which analysed the effect of structure on reading time at the main verb. There was a significant main effect of structure $\left[F_{1}(1,57)=6.42, p=.014\right.$, partial $\eta^{2}=.101 ; F_{2}(1,63)=7.46, p=.008$, partial $\left.\eta^{2}=.106\right]$, where subject relatives were processed on average $38 \mathrm{msec}$ faster than object relatives at the main verb (subject RC: $M=571.9 \mathrm{~ms}$, object RC: $M=610.2 \mathrm{~ms}$ ). This is comparable to the subject RC advantage observed in other laboratories. For instance, prior to training the control and experimental groups in Wells et al. (2009) processed the main verb in subject relatives $34 \mathrm{msec}$ and $68 \mathrm{msec}$ faster than in object relatives, respectively.

\section{Prime $x$ structure analyses}

The next set of analyses tested for the presence of a priming effect. Following Traxler and Tooley (2008), difference scores were created by subtracting the reading time of each target sentence from the reading time of each prime sentence. As such, positive difference scores indicated priming, since they suggest that processing was quicker during the target than during the prime. It was predicted that, following the frequency $x$ regularity argument, there would be a prime by target structure interaction, such that the priming effect should be greatest in the conditions where the prime sentence was an object RC. We expected this pattern of results for two reasons. Firstly, in the object RC identity prime condition we expected there to be a priming effect, such that processing an object $\mathrm{RC}$ prime eases the processing of an object RC target. Secondly, we expected the difference in processing between an object RC prime and a subject RC target to reflect the basic subject-object asymmetry observed in the prime sentences. That is, since object RCs are generally difficult in comparison to subject RCs, we expected the difference score between the object RC prime and the subject RC target in the subject $\mathrm{RC}$ opposite prime condition to be positive.

The differences scores for each condition are displayed in Table 2. Two 2 (prime: identity versus opposite) x 2 (target structure: subject versus object RC) within-subjects ANOVAs were conducted, one by participants and one by items. The main effects of prime $\left[F_{1}(1,57)<1, n s ; F_{2}(1,61)<1, n s\right]$ and target structure 
Table 2. Mean subject and object RC difference scores (in msec) (SE in brackets)

\begin{tabular}{lcc}
\hline \multicolumn{3}{c}{ Target } \\
\hline Prime & Subject RC & Object RC \\
Identity & $8.37(15.9)$ & $33.04(23.6)$ \\
Opposite & $49.06(17.6)$ & $-9.35(17.3)$ \\
Difference & -40.7 & +42.4 \\
\hline
\end{tabular}

$\left[F_{1}(1,57)<1, n s ; F_{2}(1,61)<1, n s\right]$ were not significant. The prime by target structure interaction was significant $\left[F_{1}(1,57)=5.62, p=.02\right.$; partial $\eta^{2}=.09$, $F_{2}(1,61)=4.93, p=.03$, partial $\left.\eta^{2}=.08\right]$. A simple main effects analysis showed that, consistent with our hypothesis, the conditions in which the prime was an object RC resulted in significantly higher priming $\left[F_{1}(1,57)=5.7, p=.021\right.$, partial $\eta^{2}=.09 ; F_{2}(1,63)=6.2, p=.016$, partial $\left.\eta^{2}=.09\right]$. Finally, we tested whether identity priming led to a significant facilitative effect on reading speed for object relatives in comparison to opposite priming. The difference was not significant by participants $[t(57)=1.29, p=.10]$ but was significant by items $[t(62)=2.1, p=.02]$.

\section{Eliminating the subject-object asymmetry}

The frequency $\mathrm{x}$ regularity argument predicts that the subject-object asymmetry will be reduced or eliminated following increased but equal exposure to subject and object RCs. This is because low frequency forms are predicted to benefit more from increased exposure than are forms that have comparatively higher frequency. In terms of our design, this could mean one of two things. Firstly, it could be that only immediate exposure following priming reduces the effect, which is to say that the subject-object asymmetry will only be eliminated when we compare subject and object RC targets following identity primes. Secondly, it could be that mere exposure to extra subject and objects RCs reduces or eliminates the asymmetry, which would mean that there should be no difference between target subject and object RCs following priming in general. To test the first scenario we compared reading times at the main verb in the target sentences in the two identity prime conditions (i.e., subject-subject \& object-object). The analysis showed that subject RCs were processed on average $26 \mathrm{~ms}$ faster than object RCs (subject RC: $M=$ $563.45 \mathrm{~ms}$; object RC: $M=589.48)$, a difference that was not significant by participants $\left[F_{1}(1,57)=1.91, p=.173\right.$, partial $\left.\eta^{2}=.032\right)$ and marginal by items $\left(F_{2}(1,63)\right.$ $=3.54, p=.065$, partial $\left.\eta^{2}=.053\right]$. We tested the second scenario by comparing all target subject RCs to object RCs. The analysis showed that the subject RCs were processed on average $29 \mathrm{~ms}$ faster than the object RCs at the critical main verb 
region (subject RC: $M=556.14 \mathrm{~ms}$; object $\mathrm{RC}: M=585.37 \mathrm{~ms}$ ), a difference that was significant by participants and items $\left[F_{1}(1,57)=4.6, p=.036\right.$, partial $\eta^{2}=.075$; $F_{2}(1,63)=6.3, p=.015$, partial $\left.\eta^{2}=.091\right]$. As such, it appears that the subjectobject asymmetry is most affected following identity primes.

\section{Discussion}

The present study aimed to investigate the role of prior experience in the processing of relative clause sentences using structural priming. In support of the frequency $x$ regularity interaction, Wells et al. (2009) successfully eradicated the subject-object asymmetry by training participants on relative clause structures over a period of days. In the current study we systematically investigated the effect of immediately increasing exposure to subject and object RCs using the structural priming technique. We observed that object RC primes led to a significantly greater priming effect than did subject RC primes. Part of this effect can be attributed to the fact that, in the case of the subject RC opposite prime condition, the natural subject-object asymmetry is likely to result in a positive difference score. That is, since, all things being equal, subject RCs are processed faster than object RCs, the difference score in the Object-Subject priming condition is likely to result in a positive difference score. Regardless, the object RC prime resulted in a $42 \mathrm{msec}$ improvement in the processing of object RC targets compared to when object RC targets were preceded by subject RC primes, a difference that was marginal by participants but significant by items. This result was observed in the absence of lexical overlap between prime and target, suggesting that the priming occurred at an abstract level of representation. Importantly, we also observed a significant attenuation of the subject-object asymmetry following priming. This was particularly evident immediately following identity priming, where, consistent with Wells et al., we showed that the difference in processing between subject and object RCs was not statistically significant. Indeed, following identity priming the effect size of the difference between subject and object RCs reduced threefold by participants and exactly halved by items, suggesting a sharp reduction in the complexity normally attributed to object RCs.

The results therefore suggest that, consistent with the frequency $\mathrm{x}$ regularity interaction, increasing exposure to object RCs improves the processing of these structures more than increasing exposure to subject RCs improves the processing of subject RCs. We suggest that one likely explanation for the effect concerns the mapping of participant roles to sentential positions of nouns. Gennari and MacDonald (2008) argued that much of the difficulty associated with object RCs can be attributed to syntactic and semantic indeterminacy experienced by readers as 
the sentence unfolds. That is, as an object RC unfolds there are other structural and semantic analyses that are more likely given the frequency of use in the language. For instance, there are multiple ambiguities in the fragment The lawyer that... Firstly, there is ambiguity of thematic role assignment; the lawyer could be agent, experiencer, or a patient/theme. Given that the noun is animate and human, it is more than likely that it is an agent, since animate, human NPs are generally agents. At the relative pronoun there is a structural ambiguity: the sentence could be a subject or object RC. Note that even if the subject NP is processed as a patient/ theme there is still an ambiguity, since the relative clause could be passivised, as in The lawyer that was framed by the crooked policeman. The suggestion here is that processing an object relative prime eases some of the ambiguity associated with the syntactic and semantic analysis of object relative targets. In particular, it is likely to strengthen the expectation that animate NPs in the sentence initial position can be patients/themes, and therefore promote an object RC analysis at the syntactic ambiguity experienced at the relative pronoun, easing the processing complexity traditionally experienced at the main verb.

This interpretation identifies an important role for linguistic experience in the development and implementation of parsing routines, and is therefore consistent with an approach to relative clauses where acquisition and processing represent the accumulation of statistical knowledge over the developmental history of the speaker. Such an approach is best articulated in constraint-based, connectionist, or usage-based approaches to language (e.g., Bates \& MacWhinney, 1989; Christiansen \& MacDonald, 2009; Fitz et al., this volume; MacDonald et al., 1994; Seidenberg \& MacDonald, 1999; Tomasello, 2003). On this approach, there is continuity between acquisition and processing: the adult language processing system is sensitive to the same statistical regularities that are important in language acquisition because parsing preferences are forged over the developmental history of the speaker. As we have observed in the current study, even the adult parsing system is sensitive to changes in the input, suggesting that the parser constantly updates statistical likelihoods of structural alternations.

A number of convergent strands of research provide strong evidence for the existence a learning mechanism that tracks statistical probabilities of language across the lifespan. Firstly, we have solid evidence to suggest that children in the first year of life are sensitive to the statistical properties of the ambient language (e.g., Saffran, 2003; Saffran, Newport, \& Aslin, 1996; for a review see Romberg \& Saffran, 2010). A majority of these studies test infants' ability to distinguish between pseudo-linguistic strings that either conform or do not conform to a toy grammar devoid of meaning. Recent studies have shown that children are also sensitive to the statistical properties of natural languages (Pelucchi, Hay, \& Saffran, 2009). Such studies suggest an implicit processing mechanism that can detect 
statistical regularities over formal properties of external stimuli, perhaps a mechanism akin to the simple recurrent networks (SRNs) found in connectionist models (see Chang, Dell, \& Bock 2006; Fitz et al., this volume). Several studies have now reported direct relationships between performance on statistical learning tasks and language processing. For instance, Misyak and Christiansen (2007) reported that performance on an artificial grammar learning task predicted adult's comprehension of relative clauses (see also Misyak, Christiansen, \& Tomblin, 2010). Similarly, Conway, Bauernschmidt, Huang, and Pisoni (2010) showed that performance on a visual implicit learning task significantly correlated with adult participants' ability to predict the next word in a sentence under degraded input. They concluded that their results suggest that (modality general) sequencing abilities are essential for acquiring long-term knowledge about the sequential structure of language. In a similar study conducted with children aged 4-6-years, Kidd (in press) showed that children's performance on a visual implicit learning task predicted the tendency to be primed in a structural priming task that targeted the use of the English passive structure. Interestingly, this relationship might only be related to the sequencing of word order relations, since Kidd and Kirjavainen (2011) found no relationship between implicit learning and the production of complex morphological forms in 4-6-year-old Finnish-speaking children.

A final source of evidence comes from the broader range of research on structural priming. By definition, a priming effect represents a change in linguistic behaviour as a result of experience. In fact, a well-established finding in structural priming research is that lower frequency forms tend to result in larger priming effects (Ferreira \& Bock, 2006). For instance, passives primes are more likely to lead to higher priming effects than are active primes, an effect which is captured by the frequency $\mathrm{x}$ regularity argument. One prominent explanation of priming attributes the effect largely to the statistical/implicit learning (Chang et al., 2006). Structural priming studies typically study syntactic alternations that do not differ majorly in meaning (e.g., active/passive, double object/prepositional dative). Following similar studies (e.g., Nitschke, Kidd, \& Serratrice, 2010; Traxler, 2008; Traxler \& Tooley, 2008), the results from the current study suggest that complex structures like relative clauses are subject to the same processes as observed in other structural priming studies.

In the current chapter we have presented data that suggest a prominent role for experience in the acquisition and processing of relative clauses. This experienced-based account of language does not make an artificial distinction between language acquisition and language processing; our argument is that they represent two sides of the same coin. The implication is that acquisition and processing data should mutually constrain each other, thereby moving a step closer to a more unified account of linguistic representation and performance. 


\section{References}

Bates, E. \& MacWhinney, B. 1989. Functionalism and the competition model. In The Crosslinguistic Study of Sentence Processing, B.MacWhinney \& E. Bates (eds.), 3-73. Cambridge: CUP.

Chang, F., Dell, G. S. \& Bock, J. K. 2006. Becoming syntactic. Psychological Review 113: 234-272.

Christiansen, M.H. \& MacDonald, M.C. 2009. A usage-based approach to recursion in sentence processing. Language Learning 59(Suppl. 1): 121-161.

Conway, C.M., Bauernschmidt, A., Huang, S.S. \& Pisoni, D.B. 2010. Implicit statistical learning in language processing: Word predictability is the key. Cognition 114: 356-371.

Daneman, M.E. \& Carpenter, P.A. 1980. Individual differences in working memory and reading. Journal of Verbal Learning and Verbal Behaviour 19: 450-466.

Ferreira, V.S. \& Bock, J.K. 2006. The functions of syntactic priming. Language and Cognitive Processes 21: 1011-1029.

Gennari, S.P. \& MacDonald, M.C. 2008. Semantic indeterminacy in object relative clauses. Journal of Memory and Language 58: 161-187.

Gibson, E. 1998. Linguistic complexity: Locality of syntactic dependencies. Cognition 68: 1-76.

Just, M.A. \& Carpenter, P.A. 1992. A capacity theory of comprehension: Individual differences in working memory. Psychological Review 99: 122-149.

Kidd, E. In press. Implicit statistical learning is directly associated with the acquisition of syntax. Developmental Psychology.

Kidd, E. \& Kirjavainen, E. 2011. Exploring the memory mechanisms responsible for the acquisition of past tense morphology: Evidence from Finnish. Language and Cognitive Processes 26: 794-829.

King, J. \& Just, M. A. 1991. Individual differences in syntactic parsing: The role of working memory. Journal of Memory and Language 30: 580-602.

MacDonald, M.C. \& Christiansen, M.H. 2002. Reassessing working memory: Comment on Just and Carpenter (1992) and Waters and Caplan (1996). Psychological Review 109: 35-54.

MacDonald, M.C., Pearlmutter, N.J. \& Seidenberg, M.S. 1994. Lexical nature of syntactic ambiguity resolution. Psychological Review 101: 676-703.

MacWhinney, B. \& Pleh, C. 1988. The processing of restrictive relative clauses in Hungarian. Cognition 29: 95-141.

MacWhinney, B., James, J.S., Schunn, C., Li, P. \& Schneider, W. 2001. STEP: A system for teaching experimental psychology using E-Prime. Behavior Research Methods, Instruments and Computers 33: 287-296.

Mak, W.M., Vonk, W. \& Schiefers, H. 2002. The influence of animacy on relative clause processing. Journal of Memory and Language 47: 50-68.

Mak, W.M., Vonk, W. \& Schriefers, H. 2006. Animacy in processing relative clauses: The hikers that rocks crush. Journal of Memory and Language 54: 466-490.

Misyak, J.B., \& Christiansen, M. H. 2007. Extending statistical learning farther and further: Long-distance dependencies, and individual differences in statistical learning and language. In Proceedings of the 29th annual cognitive science society conference, D. S. McNamara \& J.G. Trafton (eds), 1307-1312. Austin TX: Cognitive Science Society.

Misyak, J.B., Christiansen, M. \& Tomblin, J.B. 2010. On-line individual differences in statistical learning predict language processing. Frontiers in Psychology 1. doi: 10.3389/ fpsyg.2010.00031. 
Nitschke, S. 2010. A investigation into structural priming in comprehension in first and second language speakers. PhD thesis, The University of Manchester.

Nitschke, S., Kidd, E. \& Serratrice, L. 2010. First language transfer and long-term structural priming in comprehension. Language and Cognitive Processes 25: 94-114.

Pelucchi, B., Hay, J. \& Saffran, J. 2009. Statistical learning in a natural language by 8 - month-old infants. Child Development 80: 674-685

Romberg, A.R. \& Saffran, J.R. 2010. Statistical learning and language acquisition. Wiley Interdisciplinary Reviews in Cognitive Science. doi: 10.1002/wcs.78.

Saffran, J.R. 2003. Statistical language learning: Mechanisms and constraints. Current Directions in Psychological Science 12: 110-114.

Saffran, J., Aslin, R. \& Newport, E. 1996. Statistical learning by 8-month-old infants. Science 274: 1926

Seidenberg, M.S. 1985. The time course of phonological code activation in two writing systems. Cognition 19: 1-30.

Seidenberg, M.S. \& McClelland, J L. 1989. A distributed developmental model of word recognition and naming. Psychological Review 96: 523-568

Seidenberg, M.S. \& MacDonald, M.C. 1999. A probabilistic constraints approach to language acquisition and processing. Cognitive Science 23: 569-588

Thothathiri, M. \& Snedeker, J. 2008. Give and take: Syntactic priming during spoken language comprehension. Cognition 51: 51-56

Tomasello, M. 2003. Constructing a Language: A Usage-based Theory of Language Acquisition. Cambridge, MA: Harvard University Press.

Traxler, M.J. \& Tooley, K.M. 2008. Priming in sentence comprehension: Strategic or syntactic? Language and Cognitive Processes 23: 1-37

Traxler, M.J., Morris, R.K. \& Seely, R.E. 2002. Processing subject and object relative clauses: Evidence from eye movements. Journal of Memory and Language 47: 69-90.

Traxler, M.J., Williams, R.S., Blozis, S.A. \& Morris, R. K. 2005. Working memory, animacy and verb class in the processing of relative clauses. Journal of Memory and Language 53: 204-224

Wells, J.B., Christiansen, M.H., Race, D.S., Acheson, D.J. \& MacDonald, M.C. 2009. Experience and sentence processing: Statistical learning and relative clause comprehension. Cognitive Psychology 58: 250-271. 